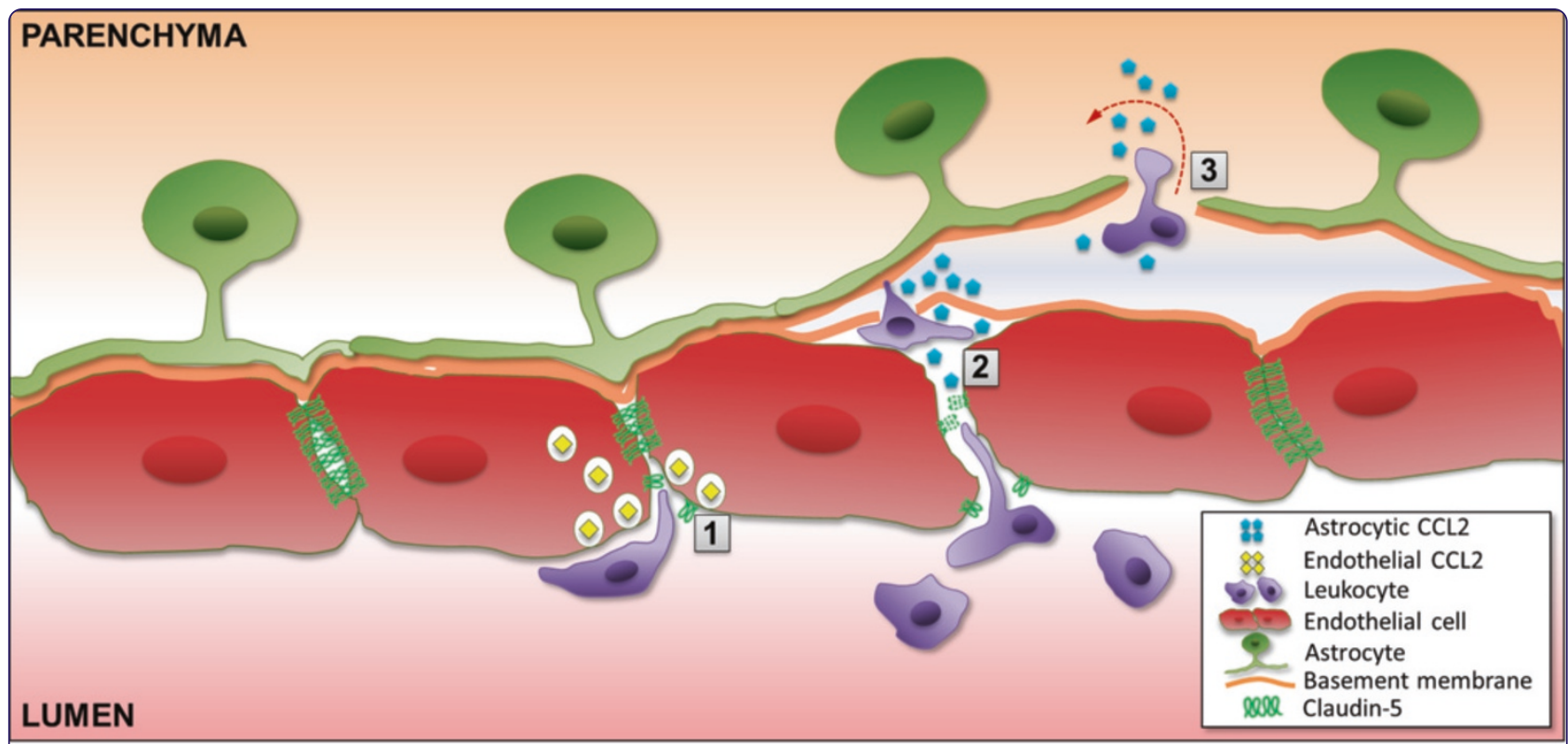

Differential actions of astrocyte-derived and endothelial cell-derived CCL2 at CNS venules

Cell-selective knockout and 3D confocal image analysis reveals separate roles for astrocyte-and endothelial-derived CCL2 in neuroinflammation

Paul et al.

C Biomed Central 


\title{
Cell-selective knockout and 3D confocal image analysis reveals separate roles for astrocyte-and endothelial-derived CCL2 in neuroinflammation
}

\author{
Debayon Paul ${ }^{1 \dagger}$, Shujun Ge ${ }^{1{ }^{*}+}$, Yen Lemire ${ }^{1}$, Evan R Jellison ${ }^{2}$, David R Serwanski ${ }^{3}$, Nancy H Ruddle ${ }^{4}$
} and Joel S Pachter ${ }^{1}$

\begin{abstract}
Background: Expression of chemokine CCL2 in the normal central nervous system (CNS) is nearly undetectable, but is significantly upregulated and drives neuroinflammation during experimental autoimmune encephalomyelitis (EAE), an animal model of multiple sclerosis which is considered a contributing factor in the human disease. As astrocytes and brain microvascular endothelial cells (BMEC) forming the blood-brain barrier (BBB) are sources of CCL2 in EAE and other neuroinflammatory conditions, it is unclear if one or both CCL2 pools are critical to disease and by what mechanism(s).
\end{abstract}

Methods: Mice with selective CCL2 gene knockout $(K O)$ in astrocytes (Astro $K O$ ) or endothelial cells (Endo $K O$ ) were used to evaluate the respective contributions of these sources to neuroinflammation, i.e., clinical disease progression, BBB damage, and parenchymal leukocyte invasion in a myelin oligodendrocyte glycoprotein peptide $\left(\mathrm{MOG}_{35-55}\right)$-induced EAE model. High-resolution 3-dimensional (3D) immunofluorescence confocal microscopy and colloidal gold immuno-electron microscopy were employed to confirm sites of CCL2 expression, and 3D immunofluorescence confocal microscopy utilized to assess inflammatory responses along the CNS microvasculature.

Results: Cell-selective loss of CCL2 immunoreactivity was demonstrated in the respective $K O$ mice. Compared to wild-type (WT) mice, Astro KO mice showed reduced EAE severity but similar onset, while Endo KO mice displayed near normal severity but significantly delayed onset. Neither of the $K O$ mice showed deficits in $T$ cell proliferation, or IL-17 and IFN- $\gamma$ production, following MOG $_{35-55}$ exposure in vitro, or altered MOG-major histocompatibility complex class II tetramer binding. 3D confocal imaging further revealed distinct actions of the two CCL2 pools in the CNS. Astro KOs lacked the CNS leukocyte penetration and disrupted immunostaining of CLN-5 at the BBB seen during early EAE in WT mice, while Endo KOs uniquely displayed leukocytes stalled in the microvascular lumen.

Conclusions: These results point to astrocyte and endothelial pools of CCL2 each regulating different stages of neuroinflammation in EAE, and carry implications for drug delivery in neuroinflammatory disease.

Keywords: Astrocytes, Blood-brain barrier, Brain microvascular endothelial cells, CCL2, Claudin-5, Central nervous system, Experimental autoimmune encephalomyelitis, Neuroinflammation, 3D confocal imaging

\footnotetext{
* Correspondence: ge@uchc.edu

${ }^{\dagger}$ Equal contributors

'Department of Cell Biology, Blood-brain Barrier Laboratory, 263 Farmington

Ave., Farmington CT 06030, USA

Full list of author information is available at the end of the article
} waiver (http://creativecommons.org/publicdomain/zero/1.0/) applies to the data made available in this article, unless otherwise stated. 


\section{Background}

The chemokine CCL2 (formerly called Monocyte Chemoattractant Protein-1, MCP-1) has long been established as a critical mediator of inflammation within and outside the central nervous system (CNS), and stimulates extravasation of mononuclear leukocytes into CNS and peripheral tissue beds [1-5]. Elevated CNS expression of CCL2 has been a consistent observation among the different paradigms of experimental autoimmune encephalomyelitis (EAE) [6-8], a CNS demyelinating inflammatory disease that serves as a model for multiple sclerosis. This chemokine's singular importance in driving EAE was demonstrated by global CCL2 knockout $(\mathrm{KO})$ mice $\left(\mathrm{CCL} 2^{-1-}\right.$ ), which showed diminished severity and delay in onset of disease in C57BL/6 mice actively immunized with myelin oligodendrocyte glycoprotein ${ }_{35-55}\left(\mathrm{MOG}_{35-55}\right)$ [9]. Adoptive transfer EAE experiments also revealed effector T cells from MOG-immunized CCL2 ${ }^{-1-}$ mice could transfer EAE to naïve wild-type ( $W T$ ) recipients, while encephalitogenic $\mathrm{T}$ cells from WT donors were unable to induce EAE in $\mathrm{CCL}^{-1-}$ mice [9]. Bone marrow chimera studies further showed active immunization EAE was markedly reduced when bone marrow from WT mice was engrafted into lethally irradiated CCL $2^{-/-}$mice, but not when bone marrow from $\mathrm{CCL} 2^{-/-}$mice was transferred into WT recipients [10]. Collectively, these findings of induced CNS expression of CCL2 during EAE, together with the adoptive transfer and bone marrow chimera studies, are consistent with a prominent role for CNS CCL2 in mediating EAE and diminish or negate the pathogenic impact of CCL2 from the peripheral leukocyte compartment.

What remains unclear, however, is which specific sources of CCL2 significantly contribute to disease, whether any reside locally in the $\mathrm{CNS}$, their pathogenic mechanisms, and how they might be targeted therapeutically. Astrocytes are a major CNS source of CCL2 in both EAE and multiple sclerosis (MS) $[7,8,11]$. By projecting their endfeet toward the abluminal surface of brain microvascular endothelial cells (BMEC) that form the blood-brain barrier (BBB), astrocytes are ideally situated to intimately modulate $\mathrm{BBB}$ function and CNS leukocyte extravasation [12]. Our laboratory [13] and others $[14,15]$ have demonstrated that CCL2 can disrupt integrity of cultured BMEC along with causing redistribution and reduction in expression of tight junction (TJ) proteins. A priori, CCL2 released from astrocyte endfeet may be partly responsible for the loss of BBB properties that accompanies both EAE [16] and MS [17], assisting development of a chemotactic gradient across the microvascular wall to drive the migration of adhered leukocytes past the endothelium, and/or further guiding extravasated leukocytes into the CNS parenchyma [18]. In addition to astrocytes, BMECs have also been shown to express CCL2 during EAE [19] and MS [20].
The observations that anti-CCL2 antibody prohibits firm attachment of leukocytes in vivo to CNS pial venules of mice immunized for EAE [21], and inhibits monocyte transendothelial migration across cultured BMEC [22], support the concept that CCL2 presented on the luminal endothelial surface aids in arresting leukocytes prior to their extravasation. The recent description that transendothelial migration of lymphocytes is mediated, in part, by intraendothelial vesicle stores of CCL2 [23], further accents a novel role for the endothelium as a critical source of this chemokine.

To resolve the respective contributions of astrocyte and endothelial cell CCL2 to neuroinflammation, we developed cell-conditional chemokine $K O$ mice, in which the CCL2 gene was selectively eliminated in each of these cell types [24]. Here we report for the first time that targeted CCL2 gene deletion from either astrocytes or endothelial cells abates EAE pathogenesis, while differentially affecting separate aspects of CNS leukocyte extravasation and clinical disease course.

\section{Materials and methods \\ Animals}

Astrocyte and endothelial cell-specific CCL2 knockout (KO) mice were generated by intercrossing mice containing a floxed CCL2 allele with transgenic mice of glial fibrillary acid protein (GFAP)-Cre or Tie2-background, respectively, and have been previously characterized in detail [24]. Astrocyte-specific $K O$ mice are referred to as Astro $K O$, and endothelial specific $K O$ mice as Endo $K O$ mice throughout this study. $K O$ mice and their wild-type (WT) littermate controls were housed in specific pathogenfree conditions. All procedures involving animals were performed in accordance with the Animal Care and Use Guidelines of the University of Connecticut Health Center(Animal Welfare Assurance \#A3471-01).

\section{EAE induction}

EAE was induced by active immunization with $\mathrm{MOG}_{35-55}$ peptide (MEVGWYRSPFSRVVHLYRNGK; W. M. Keck Biotechnology Resource Center, Yale University), as previously described [25]. Briefly, on day 0 (d0), female mice 8 to 10 weeks of age were injected subcutaneously into the right and left flanks with a total of $300 \mu \mathrm{g}$ of MOG peptide in complete Freund's adjuvant containing $300 \mu \mathrm{g} \mathrm{Myco-}$ bacterium tuberculosis (DIFCO). Mice were also injected intraperitoneally with $500 \mathrm{ng}$ pertussis toxin (List Laboratories) in phosphate buffered saline (PBS, Gibco/BRL) on d0 and $\mathrm{d} 2$ post-immunization (p.i.) to heighten the autoimmune reaction to $\mathrm{MOG}_{35-55}$ peptide [26,27].

\section{Clinical assessment of EAE}

Mice were scored daily for clinical disease severity according to the following scale: $0=$ normal; 1 = tail limpness; 
$2=\operatorname{limp}$ tail and weakness of hind legs; $3=\operatorname{limp}$ tail and complete paralysis of hind legs; $4=$ limp tail, complete hind leg and partial front leg paralysis; and $5=$ death. Several disease parameters were calculated as described [28]. The Mean Day of Onset was calculated by averaging the time when clinical symptoms; i.e., a clinical score of $\geq 1$, were first observed for two consecutive days in each mouse. The Mean Maximum Clinical Score was calculated by averaging the highest score for each mouse. The Disease Index was calculated by adding the daily average clinical scores in each group, dividing by the mean day of onset, and multiplying by 100 . If an animal showed no disease, the day of onset was arbitrarily counted as one day after the last day of the experiment. Disease Incidence was the fraction of mice experiencing EAE.

\section{Cell culture and cytokine assay}

$\mathrm{MOG}_{35-55}$-immunized mice were sacrificed and draining lymph nodes were dissected on d12. Mashed lymph nodes were pressed through a $70 \mu \mathrm{m}$ mesh into cold RPMI. Cells were pelleted at $450 \mathrm{~g}$ at $4^{\circ} \mathrm{C}$ for $5 \mathrm{~min}$ and resuspended in red blood cell lysis buffer (Sigma) on ice for $5 \mathrm{~min}$. After three washes with cold PBS, cells were stained with $0.4 \%$ Trypan blue (Sigma Aldrich) and counted with the Countess ${ }^{\circ}$ Automated Cell Counter (Invitrogen) to permit discrimination of dead cells.

Single cell suspensions of lymph node cells (LNCs) were prepared and cultured in 24-well plates (Corning) at $1 \times 10^{6}$ viable cells $/ \mathrm{mL}$ in RPMI 1640 supplemented with $10 \%$ fetal bovine serum, $1.25 \%$ HEPES buffer, $1 \%$ sodium pyruvate, $1 \%$ penicillin-streptomycin, $1 \%$ glutamine, $1 \%$ non-essential amino acids, $0.01 \% 0.05 \mathrm{M}$ 2-mercaptoethanol (Sigma Aldrich). LNCs were restimulated with a combination of $10 \mu \mathrm{g} / \mathrm{mL} \mathrm{MOG}_{35-55}$ and $0.5 \mathrm{ng} / \mathrm{mL}$ interleukin-12 (IL-12) (R\&D Systems). Cytokines present in the cell culture supernatants of LNC were quantified using the multiplex enzyme-linked immunosorbent assay (ELISA) kit (R\&D Systems).

\section{Proliferation assay}

LNCs were prepared as for the cytokine assay. Cells were pulse-labeled with $2 \mu \mathrm{M}$ carboxyfluoresceinsuccinimidyl ester (CFSE, Molecular Probes) in RPMI for 5 min at room temperature. After extensive washing with PBS, the CFSE-labeled cells were suspended and cultured in complete medium, as above, in 24-well plates $\left(2 \times 10^{6} \%\right.$ well) for $72 \mathrm{~h}$. Cell viability was assessed by Trypan blue exclusion. The LNC samples were washed in fluorescenceactivated cell sorting (FACS) buffer (1\% FCS and 0.1\% sodium azide in PBS). After blocking with $\mathrm{F}_{\mathrm{c}}$ Block (BD Biosciences) at $4^{\circ} \mathrm{C}$ for $20 \mathrm{~min}$ in the dark, the cells were washed and stained with fluorochromelabeled antibodies against murine CD3, CD4, and CD11a (BD Biosciences) at $4^{\circ} \mathrm{C}$ for $30 \mathrm{~min}$. After the cells were washed, the fluorescence intensities were measured by a FACS LSRII flow cytometer (BD Biosciences) and the following parameters were determined using FlowJo software (Treestar): \% divided (percentage of cells in original population that has divided); Division Index (average number of cell divisions that a cell in the original population has undergone); Proliferation Index (total number of divisions/number of cells that went into division); Expansion Index (fold-expansion of overall culture); and Replication Index (fold-expansion of only responding cells).

\section{$\mathrm{MOG}_{38-49}$ MHC class II tetramer binding assay}

The MOG $_{38-49}$ MHC class II tetramer binding assay was based on that of Cravens et al. [29]. LNCs from $\mathrm{MOG}_{35-55^{-}}$ immunized mice were prepared and cultured as for cytokine and proliferation assays for $72 \mathrm{~h}$. Thereafter, LNCs were washed with FACS buffer, and blocked with $F_{c}$ Block in FACS buffer at $4^{\circ} \mathrm{C}$ for $20 \mathrm{~min}$ in the dark. LNCs were washed again, and $\mathrm{MOG}_{38-49}$ major histocompatibility complex (MHC) class II tetramer-PE or MHC class II control tetramer-PE hCLIP $103-117-\mathrm{PE}$ (obtained from the $\mathrm{NIH}$ Tetramer Core Facility) were added and incubated at $37^{\circ} \mathrm{C}$ for 1 hour in the dark. After incubation, the cells were directly stained with fluorochrome-labeled antibodies against murine CD4, CD11a, and CD44 at $4^{\circ} \mathrm{C}$ for $30 \mathrm{~min}$. LNCs were washed and resuspended in FACS buffer. Samples were acquired on a FACS LSRII flow cytometer (BD Biosciences) and the data analyzed using FlowJo software (Treestar). Cells were gated as single live $\mathrm{CD} 4^{+} \mathrm{T}$ lymphocytes and examined for CD11a, CD44, and tetramer reactivity. Cells that were MOG tetramerpositive and $\mathrm{CD} 11 \mathrm{a}^{+}$were considered MOG-specific and previously activated.

\section{Immunofluorescence and 3D analysis of confocal z-stack images}

Tissue was prepared as described by Paul et al. [30]. In brief, following transcardiac perfusion/fixation of mice, spinal cords were removed by laminectomy and freezeembedded in cryomatrix. Subsequently, $12 \times 60 \mu \mathrm{m}$ cryosections from the thoraco-lumbar region, approximately between the T10 and L3 vertebrae were adhered to polyL-lysine coated slides. Following staining, sections were mounted in $\mathrm{Mowiol}^{\circ}$ prior to microscopic analysis.

For immunodetection of CCL2, affinity-purified rabbit anti-mouse CCL2 (Peprotech) was utilized with a corresponding anti-rabbit Alexa ${ }^{\circ}$ 555-conjugated antibody (Life Technologies). Sections were subsequently doubleimmunostained with either rat anti-mouse CD31 (BD Pharmingen) followed by secondary incubation with anti-rat Alexa 488 (Life Technologies) to highlight the endothelium, or Alexa ${ }^{\circ} 488$-conjugated anti-mouse GFAP (Life Technologies) to identify astrocytes. To specifically enhance detection of CCL2, which shows dispersed 
punctate immunoreactivity, confocal z-stacks were first deconvolved using AutoQuant $\times 3$ (Media Cybernetics) software to correct for $\mathrm{z}$-axis distortion. This significantly improved z-resolution and the resulting high-resolution images were exported into Imaris ${ }^{\oplus}$ (Bitplane Inc.). Representative z-slices, showing the co-localization of CCL2 with endothelial CD31 or astrocyte marker GFAP were then obtained.

For 3D quantification of microvascular tight-junction protein claudin-5 (CLN-5) density, the protocol recently detailed by Paul et al. [30] was used. The microvascular basement membrane (BM), a fusion of the respective endothelial and parenchymal BMs [31], was labeled with rabbit anti-mouse Laminin 1 (Lam 1) (Cedarlane) and anti-rabbit Alexa 555 (Life Technologies). Anti-mouse Claudin-5-Alexa 488 (Life Technologies) was employed to highlight TJs. Additionally, nuclear stain DRAQ5 (Biostatus Ltd.) was utilized to reveal the perivascular cellularity surrounding inflamed CNS microvessels, identified as venules [30], due to leukocyte extravasation.

Spinal cord venules from comparable regions of the dorsolateral white matter were imaged. Confocal z-stacks were acquired at 1- $\mu \mathrm{m}$ increments between $\mathrm{z}$-slices, following a multitrack scan, using a Zeiss LSM 510 Meta confocal microscope equipped with a 40× Fluar (NA 1.3) and a $63 \times$ Plan-neofluar (NA 1.25) oil immersion lens. Confocal z-stacks were imported into Imaris ${ }^{\oplus}$ (version 7.6) software (Bitplane Inc.) and the venule of interest was segmented out from rest of the 3D dataset by manually tracing the vessel contour in each confocal $\mathrm{z}$-slice, followed by merging the $\mathrm{z}$-slice contours into a $3 \mathrm{D}$ contour surface. Surface area of the generated 3D contour was used as an estimate of the microvascular "surface area" defined by the endothelial layer. The CLN-5 channel was isosurface rendered (within the $3 \mathrm{D}$ contour surface) and the density of CLN-5 staining calculated as Total CLN-5 intensity/ Microvascular surface area. CLN-5 density values were expressed as mean \pm standard error of the mean (SEM).

To optically isolate (3D segmentation) and resolve the DRAQ $5^{+}$cellularity associated with leukocyte accumulation in the luminal or perivascular compartments of an inflamed venule [30], confocal z-stacks of venules revealing a cross-sectional view were acquired and imported into Imaris ${ }^{\ominus}$. The venule of interest was then isolated from rest of the $3 \mathrm{D}$ dataset by creating a $3 \mathrm{D}$ contour surface, defining the parenchymal BM. This effectively eliminated the parenchymal cellularity (i.e., extravascular infiltrates). Additionally, the lumen was segmented out in a similar manner, by creating another $3 \mathrm{D}$ surface, approximating the contour of the endothelial BM (Endo $\mathrm{BM})$. Spatial location of the observed cellularity between the BMs was considered perivascular (Additional file 1).

To graphically resolve the distribution of DRAQ $5^{+}$luminal and perivascular cells along microvascular $\mathrm{x}, \mathrm{y}$, and $\mathrm{z}$ axes with respect to the endothelial and parenchymal BMs, a 3D volume was first constructed from the acquired confocal z-stack, followed by $3 \mathrm{D}$ segmentation of the luminal and perivascular compartments as described above. Imaris ${ }^{\oplus}$ spot creation wizard was then employed to represent each of the $\mathrm{DRAQ}^{+}$nuclei as a "spot object" in 3D space. Only DRAQ $5^{+}$nuclei $>3 \mu \mathrm{m}$ in diameter were considered. The 3D profiles of the created spot objects, representing the position of luminal and perivascular leukocytes, were then plotted on a 3D Imaris $^{\oplus}$ Vantage plot to reveal its spatial location.

\section{Immuno-electron microscopy (Immuno-EM)}

Colloidal gold detection of CNS microvascular CCL2 immunoreactivity by immuno-EM was performed as described previously [32]. WT mice at d16 EAE were anesthetized as described above, and subjected to transcardiac perfusion/fixation with Ringer's solution, $\mathrm{pH}$ 6.9, followed by $4 \%$ paraformaldehyde, $0.1 \%$ glutaraldehyde in $0.1 \mathrm{M}$ phosphate buffer (PB), $\mathrm{pH}$ 7.4. Vibratome sections of spinal cord (300-500 $\mu$ m thick) were cryoprotected with $2 \mathrm{M}$ sucrose in $\mathrm{PB}$ and plunge-frozen in liquid propane cooled by liquid nitrogen $\left(-186^{\circ} \mathrm{C}\right)$. Sections were stained en bloc with $1.5 \%$ uranyl acetate in anhydrous methanol at $-90^{\circ} \mathrm{C}$ for 30 hours and infiltrated with Lowicryl HM20 resin (Polysciences), followed by polymerization with UV light for 72 hours in a freeze-substitution instrument (Leica AFS) in a temperature gradient $\left(-45^{\circ} \mathrm{C}\right.$ to $\left.0^{\circ} \mathrm{C}\right)$. Sections $(70-80 \mathrm{~nm}$ thick) were cut and collected onto 400-mesh gold-gilded nickel grids coated with a Coat-Quick "G" pen (Daido). Tissue sections were incubated with anti-mouse CCL2 antibody (Peprotech), followed by incubation with goat anti-mouse IgG labeled with colloidal gold particles of $12 \mathrm{~nm}$ diameter (Jackson ImmunoResearch). After immunoreaction, tissue sections were counterstained with $2 \%$ uranyl acetate and then with $2 \%$ lead citrate. Primary antibody was omitted in immunoreaction as a control, and yielded no detectable gold labeling.

\section{Statistical analysis}

For analysis of cell-specific $K O$ on clinical EAE parameters, a $\chi^{2}$ test was used for comparisons of disease incidence; a Mann-Whitney U-test was used for comparisons of disease severity; and ANOVA, followed by Bonferroni's multiple comparison post-hoc analysis, were used for comparison of disease onset [33]. ANOVA/Bonferroni post-hoc tests were also employed to assess differences in CLN-5 density values [30]. To contrast the rate of rise of clinical EAE progression among the different mouse groups, linear regression was performed on data points beginning at the onset of disease through attainment of the plateau or highest score. Statistical analyses were 
performed employing Prism 5 software (GraphPad), and results were considered significant at $P \leq 0.05$.

\section{Results}

\section{Astro $\mathrm{KO}$ and Endo $\mathrm{KO}$ mice show cell-selective loss of} CCL2

Immunofluorescent staining of CCL2 in spinal cord during EAE in Astro KO, Endo $\mathrm{KO}$, and $W T$ mice is shown in high-resolution z-stack confocal images in Figure 1. Using identical image acquisition parameters, no CCL2 staining was detected either in naïve mice, or EAE mice in the absence of primary antibody (Additional file 2: Figure S1), thus highlighting specific immunoreactivity to inflamed CNS tissue. In WT mice at d16 post-EAE induction, CCL2 staining was vessel-associated as well as within the perivascular space (Figure 1a). Notably, CCL2 staining appeared aligned with inter-endothelial junctions (CD31), and showed a punctate distribution rather than a diffuse appearance throughout the cytoplasm. This could reflect containment of CCL2 within vesicles, as recently described by Shulman et al. [23] for cultured human umbilical vein endothelial cells (HUVECs). A representative $z$-slice further revealed intense double staining (CCL2 and CD31) of the endothelial layer. Parenchymal CCL2 staining (Figure 1b) was largely observed in association with $\mathrm{GFAP}^{+}$astrocytes. Localization within astrocytes was confirmed in a representative z-slice. Some large deposits of CCL2 could also be seen just outside the astrocytes, which may indicate secreted chemokine. Both endothelial and astrocyte immunoreactivity in WT mice with EAE were confirmed by immuno-electron microscopy (Figure 1c-f). Notably, CCL2 immunoreactivity was detected within the inter-endothelial junctions (Figure 1c), and could also be seen in association with endothelial vesicular-like structures (Figure 1d). There was also abundant immunoreactivity within cellular processes at the abluminal side of the endothelium, possibly representing CCL2 destined for or contained within astrocyte endfeet (Figure 1e-f).

The CCL2 staining patterns with both $K O$ mice were markedly different (Figure 2). Astro $K O$ mice at d16 (Figure 2a) showed vessel-associated CCL2 staining but greatly diminished staining in the perivascular space. Conversely, Endo KO mice (Figure 2b) exhibited a near absence of vessel-associated CCL2 staining, while robust $\mathrm{CCL}_{2}{ }^{+}$astrocytes were still clearly evident. These staining patterns are consistent with previous results from this laboratory showing CCL2 RNA expression by both microvessel and parenchymal fractions of brain and spinal cord from WT mice with EAE [33], and argue that CCL2 protein is produced by astrocytes and BMECs, and not merely taken up at these sites.

That some vessels in Astro KO mice and some astrocytes in Endo KO mice appeared devoid of CCL2 staining possibly reflects that not all of the respective endothelial cell and astrocyte populations became similarly inflamed, a prospect supported by only a subset of vessels/ vessel segments being associated with leukocyte infiltrates (data not shown). It may further be that CCL2 from endothelial cells or astrocytes exerts some positive control over CCL2 expression by the other cell type [24,34].

\section{Astro $K O$ and Endo $K O$ mice are both resistant to EAE, but show different clinical phenotypes}

Figure 3a,b shows that targeted CCL2 gene eliminations in astrocytes and endothelial cells, respectively, prominently affected development of EAE, consistent with earlier reports of the effects of global CCL2 knockout $[9,10]$. While the incidence of disease was largely unaffected in both $K O$ mice compared to $W T$, there was clear evidence of altered disease progression in the two cellselective CCL2 KO groups. Astro KO mice exhibited significantly reduced disease severity (clinical score) compared with WT littermates throughout the time frame observed, along with just a slight, insignificant delay in disease onset. In sharp contrast and nearly mirror image effect, Endo KO mice showed only mild reduction in disease severity, but a significantly protracted delay in the onset of disease. It was further observed that the rate of rise of clinical disease, as reflected by the slope of the linear, ascending region of the disease score graphs, was different between Astro $K O$ and Endo KO mice. Astro KO mice showed a lesser rate of rise of clinical disease than both WT and Endo $K O$ mice, while the latter two mouse groups showed similar rates of disease rise. Thus, it appears that while disease was significantly delayed in Endo $K O$ mice, once disease commenced it proceeded on a normal time course (for the period evaluated). Comparisons of Disease Incidence, Mean Day of Onset, Mean Maximum Clinical Score, and Disease Index in Astro KO, Endo $K O$, and $W T$ mice are tabulated in Figure 3c.

\section{Astro KO and Endo KO mice do not show different MOG-specific $T$ cell responses}

Endo $K O$ mice have the CCL2 gene eliminated from all endothelial cells, central and peripheral. This could mean that observed alterations in EAE disease progression in Endo $K O$ mice might stem from a defect in the afferent immune arm, such as attenuated $\mathrm{T}$ cell priming. Indeed, this possibility is prompted by findings that CCL2 is expressed and presented by high endothelial venules in lymph nodes [35], instrumental in dendritic cell maturation [36], and released by dendritic cells to attract antigen-specific $\mathrm{T}$ cells [37]. Hence, several $\mathrm{T}$ cell proliferation parameters - \% Divided, Division Index, Proliferation Index, Expansion Index, and Replication Index - were assayed in CSFE pulse-labeled LNC cultures from 


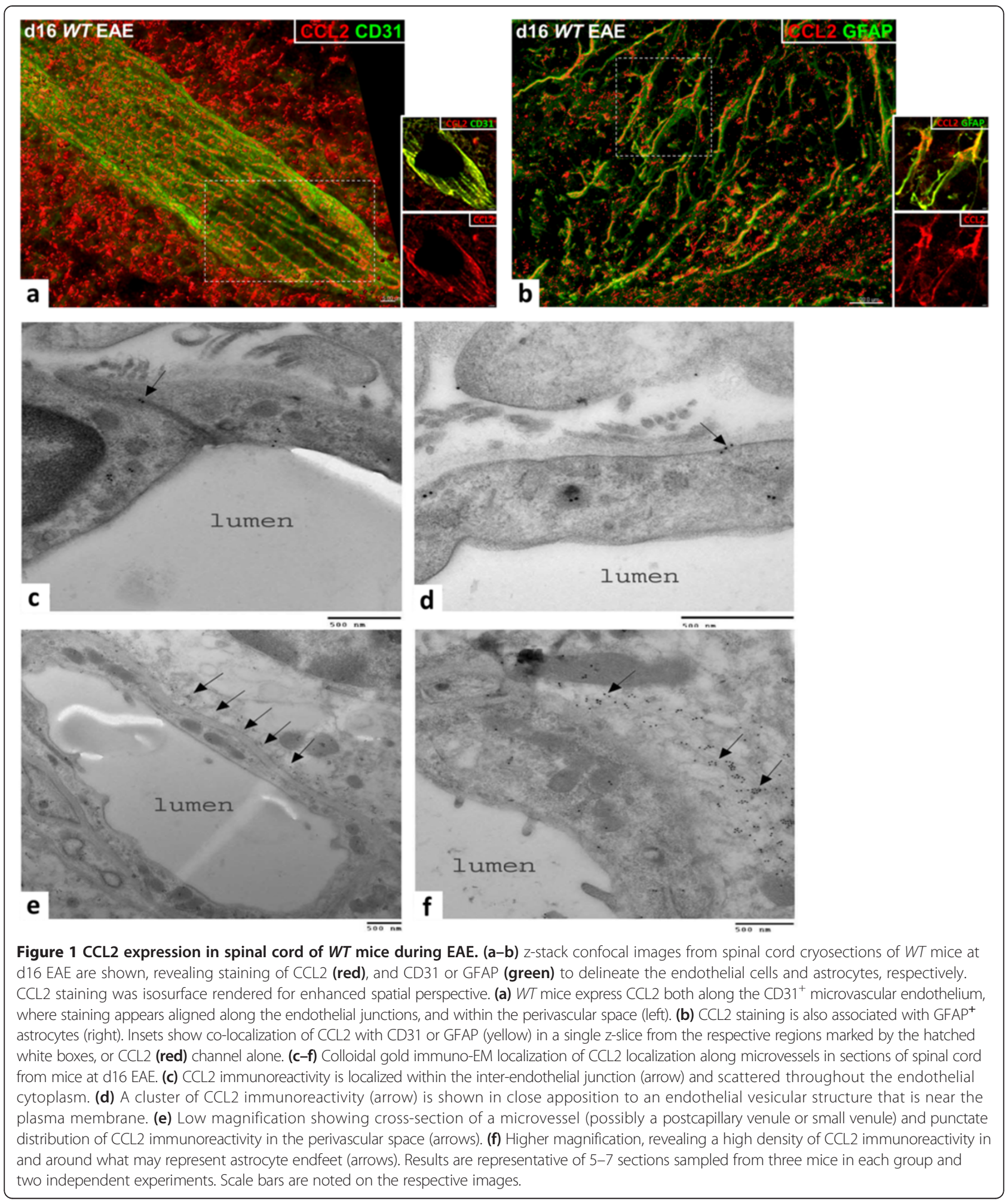

MOG-immunized Astro KO, Endo KO, and WT mice (Figure 4a). Following restimulation with MOG, no significant differences were detected among cells from the three types of mice for any of these parameters. This is in agreement with previous findings that indicated no difference in $\mathrm{T}$ cell proliferation between $\mathrm{LNC}$ cultures from $W T$ and global CCL2 $2^{-/-}$mice [9].

We also assayed the ability of cultured LNCs from each of these mice to produce IFN- $\gamma$ or IL-17, which are cytokines considered instrumental in autoimmunity and 

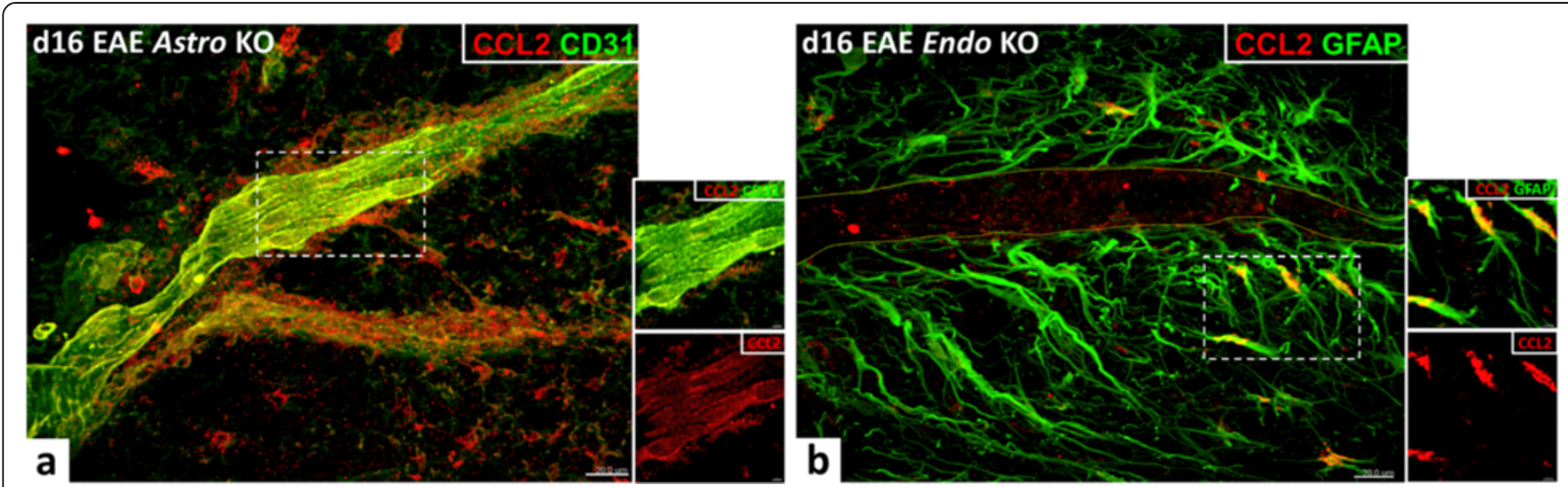

Figure 2 CCL2 expression in spinal cord of Astro $K O$ and Endo $K O$ during EAE. Representative $z$-stack confocal images from spinal cord cryosections of $K O$ mice at d16 EAE are depicted. Cell-specific CCL2-KO mice display loss of CCL2 staining in respective targeted cell types. (a) Astro KO mice show venule-associated CCL2 staining, but lack staining in the parenchymal astrocytes (left). (b) In contrast, Endo KO mice are deficient in vessel-associated CCL2 staining, but maintain astrocyte staining (right). The endothelial boundary is marked with yellow lines. Insets show co-localization of CCL2 with CD31 or GFAP (yellow) in a single z-slice from the respective regions marked by the hatched white boxes, or CCL2 (red) channel alone. Results are representative of 5-7 sections sampled from three mice in each group and two independent experiments.

EAE [38], in response to restimulation with MOG. Figure 4b shows that MOG-stimulated expression of neither cytokine was significantly altered in Astro KO or Endo KO mice compared to their WT cohorts. Earlier, Huang et al. [9] had described diminished production of
IFN- $\gamma$ in LNCs from CCL2 ${ }^{-/}$mice, which might reflect the effects of having depleted a peripheral CCL2 pool distinct from that of endothelial cells. Figure 4c further reveals that, following MOG immunization, the percentage of $\mathrm{MOG}_{38-49} \mathrm{MHC}$ class II tetramer positive CD4 T cells
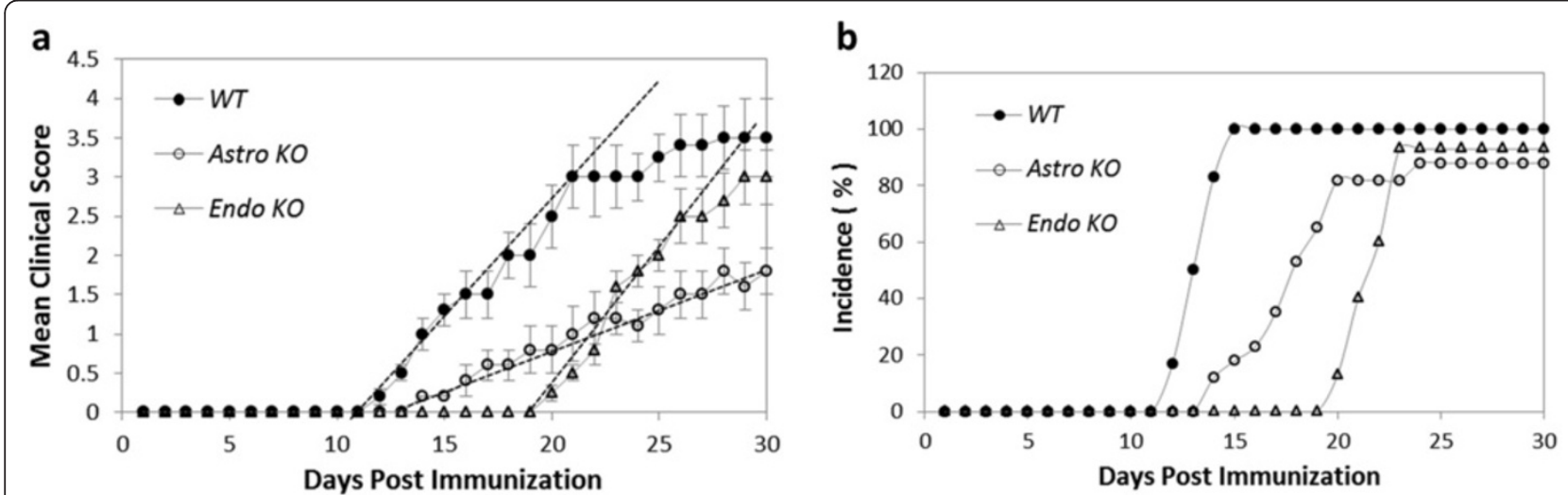

C

Summary of effects of Astro $K O$ and Endo $K O$ on clinical EAE paragraph

\begin{tabular}{|c|c|c|c|c|}
\hline Group & Incidence & Mean day of onset ${ }^{a}$ & Mean max clinical score & Disease index ${ }^{b}$ \\
\hline$W T$ & $18 / 18$ & $13.6 \pm 1.82$ & $3.0 \pm 0.77$ & 385 \\
\hline Astro KO & $15 / 17$ & $17.6 \pm 2.1$ & $1.3 \pm 0.67^{* *}$ & 115 \\
\hline Endo KO & $14 / 15$ & $22.1 \pm 3.41 * *$ & $2.0 \pm 0.46 *$ & 97 \\
\hline
\end{tabular}

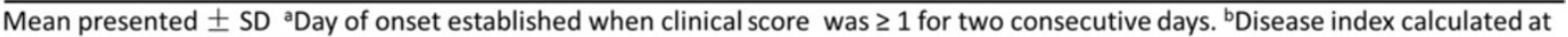
day $30 .{ }^{*} p \leq 0.05 * * p \leq 0.01$, when compared with $W T$.

Figure 3 Astro $K O$ and Endo $K O$ mice show different patterns of clinical EAE. EAE was induced in WT, Astro KO, and Endo KO mice by $M_{35}$ immunization; all mice were observed daily and scored for clinical disease for 30 days. Each group consisted of 5-6 mice, and analysis was performed in triplicate. Graphs represent mean data points from all three analyses \pm standard error. (a) Mean clinical EAE scores. Astro KO mice do not attain as severe disease as WT during the evaluation period, while Endo KO mice approach WT disease severity but do so only after significantly delayed onset. The rate of rise of clinical disease, as reflected by the slope of each regression line (hatched lines) through the respective ascending disease scores for the different mice, is similar in both WT and Endo KO mice, but notably less in Astro KO mice. (b) Disease incidence. All mice show a similar incidence of disease but, compared to WT mice, Astro KO mice show only a mild delay while Endo KO mice show a prolonged delay in disease onset. (c) Summary of various clinical disease parameters among the three mouse groups. 
was in line with another report [29] and similar in LNCs from Astro $K O$, Endo $K O$, or WT mice, indicating that the frequency of MOG-specific T cells was comparable among these groups. Hence, our results argue against either astrocyte- or endothelial cell-targeted CCL2 gene deletion having significantly impaired peripheral $\mathrm{T}$ cell behavior and immune responsiveness to MOG peptide. These findings are further in accord with reports that MOG-specific, encephalitogenic $\mathrm{T}$ cells can be generated in mice with global knockout of CCL2 [9].

Astro $K O$ and Endo KO mice display altered inflammatory responses along the CNS microvasculature during EAE Given the different clinical EAE phenotypes of Astro KO and Endo KO mice with apparent absence of overt impact on $\mathrm{T}$ cell priming, we next sought to determine effects of cell selective CCL2 loss on inflammatory events along the CNS microvasculature. $3 \mathrm{D}$ perspective projection views of confocal reconstructions were generated using Imaris ${ }^{\oplus}$ to provide a more realistic $3 \mathrm{D}$ representation of the z-stack dataset. Notable differences in staining of CLN-5, a prominent TJ protein in CNS microvessels and BBB determinant [39], were found among WT, Astro KO, and Endo $K O$ mice during EAE (Figure 5), while naïve mice of all groups showed no evidence of disparities (data not shown). At $\mathrm{d} 9$, prior to evidence of clinical disease, WT mice showed focal fragmentation of the CLN- 5 staining pattern. This discontinuous appearance of CLN-5 staining in regions of increased perivascular cellularity was not a result of CLN-5 staining being distributed in z-planes not captured during acquisition, as the confocal reconstructions shown represent 3D images generated from $60 \times 1-\mu \mathrm{m}$ thick z-slices. Rather, the areas lacking immunostaining reflected actual sites of CLN-5 disruption associated with extravasating leukocytes. This picture differed significantly from that seen with naïve mice, where CLN-5 immunostaining 


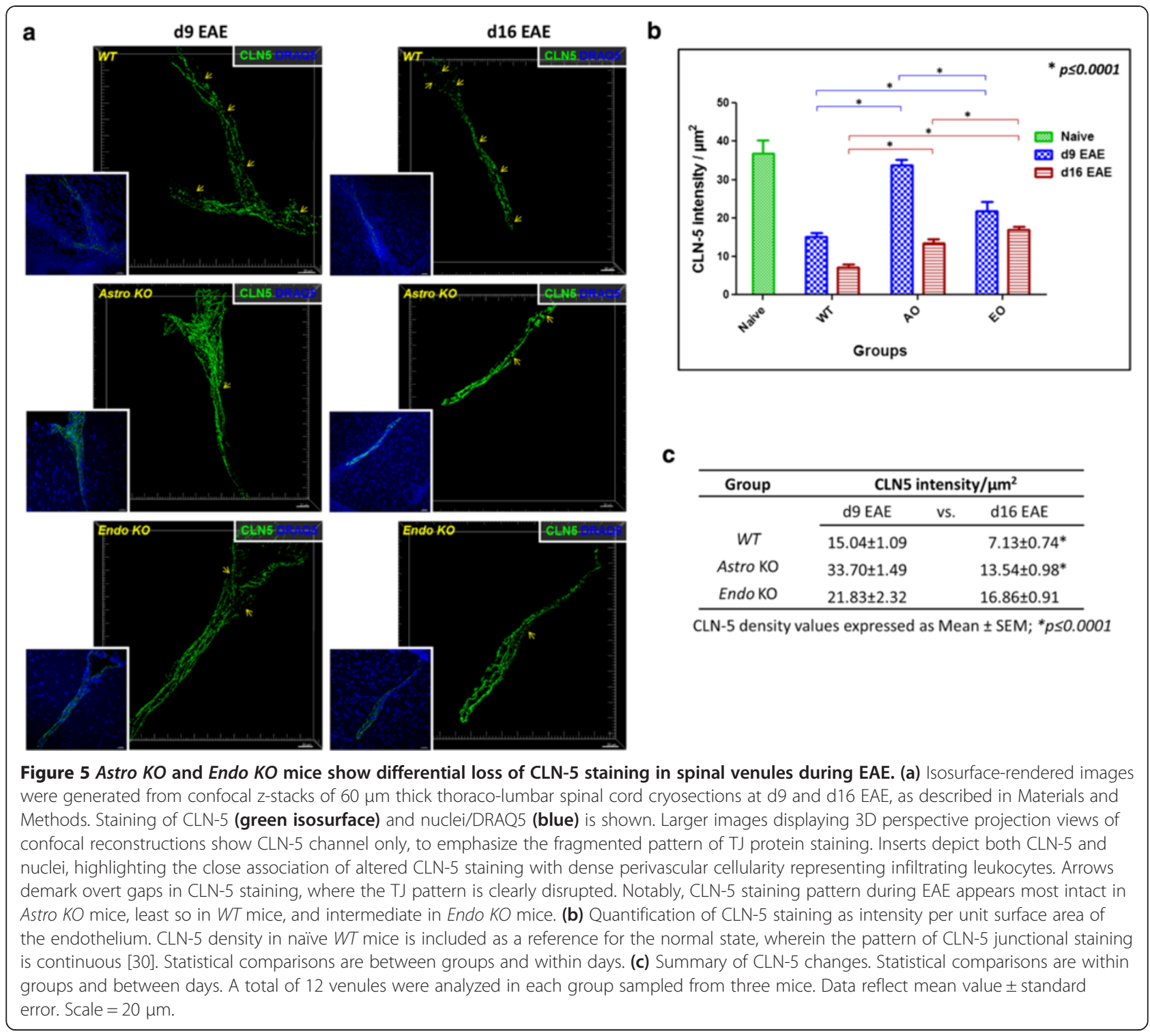

appeared continuous, without obvious interruptions, and perivascular cellularity was absent (Additional file 3: Figure S2). Hence, for the purpose of highlighting the pathologic role(s) of CCL2 released from astrocyte and endothelial sources in neuroinflammation, statistical comparisons for CLN-5 density were only made between WT, Astro KO, and Endo KO mice during evolving EAE. The significantly altered CLN-5 pattern observed in WT mice during EAE corresponded with a sharp decrease of approximately $60 \%$ in the density of CLN-5 staining. In stark comparison, Astro $\mathrm{KO}$ mice demonstrated little if any change in CLN-5 staining, while Endo $\mathrm{KO}$ mice displayed a somewhat intermediate response at this time-point. By $\mathrm{d} 16$ - following disease onset- WT mice showed further disruption and loss of CLN-5 staining, down approximately $80 \%$ from that demonstrated by naïve mice. Astro $K O$ mice at this later time showed a precipitous loss of CLN-5 staining, declining by approximately $60 \%$ (compared to naïve and d9 mice), while Endo $K O$ mice at d16 showed only a moderate, non-significant CLN-5 decrease of approximately 19\% (compared to d9).

Differences in leukocyte infiltration patterns were also obvious among the various strains of mice when vessels were viewed in longitudinal- and cross-section (Figure 6). During neuroinflammation, the BM splits into its respective endothelial and parenchymal components [31], highlighting two spaces: the subendothelial space (between the endothelium and endothelial BM) and the perivascular space (between the endothelial BM and the parenchymal BM). Both these spaces swell with leukocytes that have recently extravasated across the BMECs, 


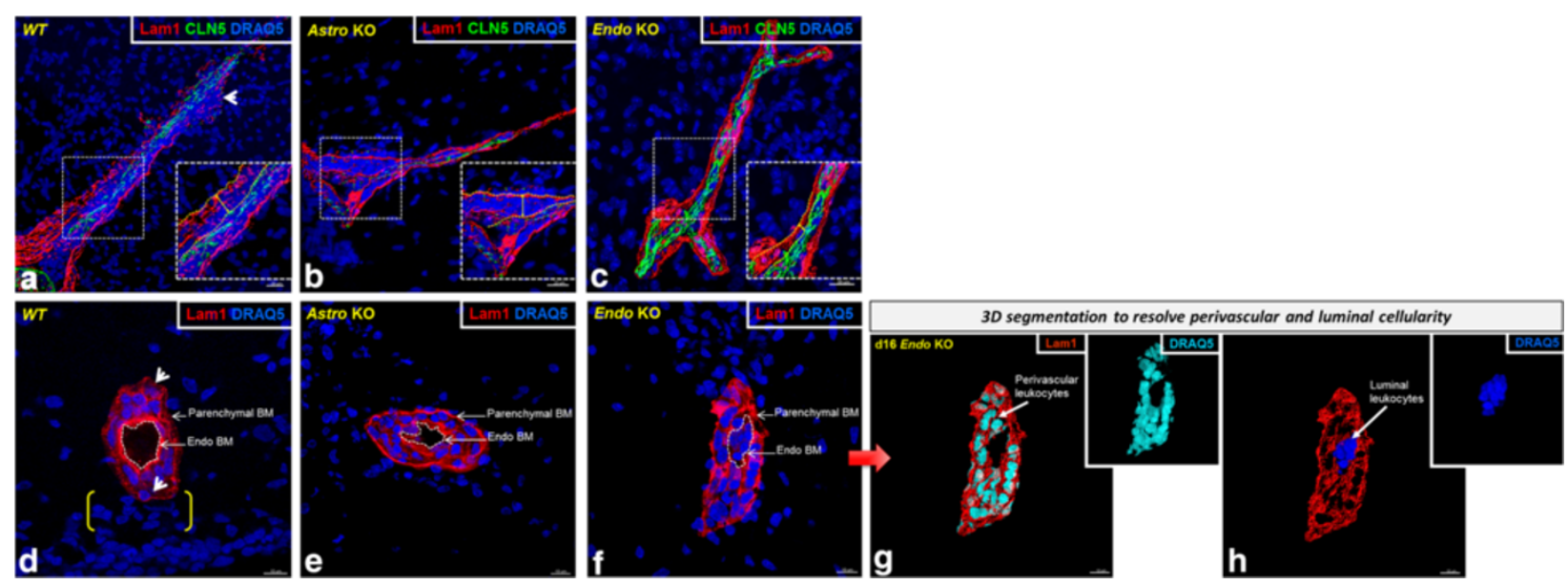

Figure 6 Astro KO and Endo KO mice display differences in perivascular cellularity associated with spinal venules during EAE. Isosurface-rendered images were generated from confocal z-stacks of 60 m cryosections at d16 EAE. Staining of BM Lam 1 (red), CLN-5 (green), and nuclei/DRAQ5 (blue) is shown. (a, b, c) Longitudinal sections reveal the extent of vessel-associated leukocytes. CLN-5 staining is presented to highlight the endothelial boundary. Insets represent enlarged view of areas highlighted in white hatched boxes, while double-headed arrows denote the space between the endothelial and parenchymal BMs. All extravasated leukocytes within this space are considered "perivascular". In WT mice, a dense accumulation of DRAQ5 ${ }^{+}$perivascular cells (representing leukocytes) is seen, a few apparently penetrating the fragmented parenchymal BM (arrowhead). In Astro KO mice, a similar dense perivascular cellularity is observed, with visibly intact parenchymal BM and lack of parenchymal leukocyte migration. In Endo $K O$ mice, the BM is also apparently intact, with minimal perivascular cellularity. Scale $=20 \mu \mathrm{m}$. (d, e, f) Cross-sections highlight the spatial distribution of vessel-associated leukocytes. In WT mice, the vessel lumen (demarked by white dashes) appears empty and cells are seen in the perivascular space. A few cells are visibly penetrating the parenchymal BM (arrowheads), alongwith dense parenchymal cellularity (brackets). In Astro KO mice, the lumen again appears empty; congregated cells are evident in the perivascular space, with a few parenchymal clusters. In Endo KO mice, cells are clearly present in the lumen, with apparently fewer cells in the perivascular space as compared to WT and Astro KO mice. Parenchymal clustering is seemingly absent. The diffuse DRAQ5 $5^{+}$cells are likely parenchymal neural cells. (g-h) The red arrow designates the same Endo KO image subject to contour-based 3D segmentation (see Materials and Methods) to further resolve luminal (blue) from perivascular (turquoise) cells. Results are representative of 5-6 microvessels sampled from three mice in each group and two independent experiments. Scale $=10 \mu \mathrm{m}$.

but as the BM becomes extensively fragmented during this process, demarcation between the spaces is blurred in longitudinal sections. Thus, in referring to leukocyte distribution, the term "perivascular" is used herein to describe all extravasated leukocytes that are vessel-associated. At d16, dense perivascular infiltrates were seen to be associated with venules in WT mice, with cells apparently penetrating the parenchymal $\mathrm{BM}$ to enter the parenchyma (Figure 6a,d). Astro $K O$ mice showed similar type clusters of perivascular cells, but no clear evidence of leukocytes in the act of rupturing the parenchymal BM (Figure 6b,e). Venules of both WT (Figure 6d) and Astro KO (Figure 6e) further exhibited lumens within which no cells were detectable. By contrast, Endo KO mice alone displayed aggregated cells apparently stalled in the lumen, as well as a seemingly lesser extent of perivascular cells (Figure 6c,f-h). To further resolve the aggregated cells throughout the microvascular lumen of an entire 60 $-\mu \mathrm{m}$ section from Endo $K O$ mice, and graphically distinguish this pattern from that in WT and Astro KO mice, the distribution profiles of cells associated with the lumen and perivascular space, respectively, were mapped in 3D (Figure 7). Intra-luminal cells could only be detected in Endo KO mice (Figure 7c). As the actual number of spinal vessels showing any evidence of inflammation was extremely low in Endo $K O$ mice at this time, the few examples detected showed this common appearance of hindered leukocyte migration. Moreover, since tissue was perfusion-fixed, these luminal cells are not likely to have resulted from blood stasis but, instead, suggest a possible deficit in extravasation from CNS microvessels in Endo KO mice.

\section{Discussion}

While the critical role of CCL2 in EAE has been revealed in global CCL2 knockout studies [9], and the chemokine sources mediating this effect are suggested to reside in the CNS [10], the identities of the sources responsible for specific neuroinflammatory events, e.g., effects at the BBB and leukocyte penetration into the CNS, have not been resolved. In the present study, we used mice with targeted CCL2 gene deletion in astrocytes or endothelial cells, along with 3D confocal imaging, to establish - for the first time - that CCL2 from each of these sources regulates different aspects of neuroinflammation and EAE course. Astro $K O$ mice exhibited a similar onset but reduced severity of disease compared to WT, while Endo $K O$ mice displayed nearly the opposite clinical pattern. However, neither of these $K O$ mice showed any significant changes in MOG-specific $\mathrm{T}$ cell responses in LNC cultures, 


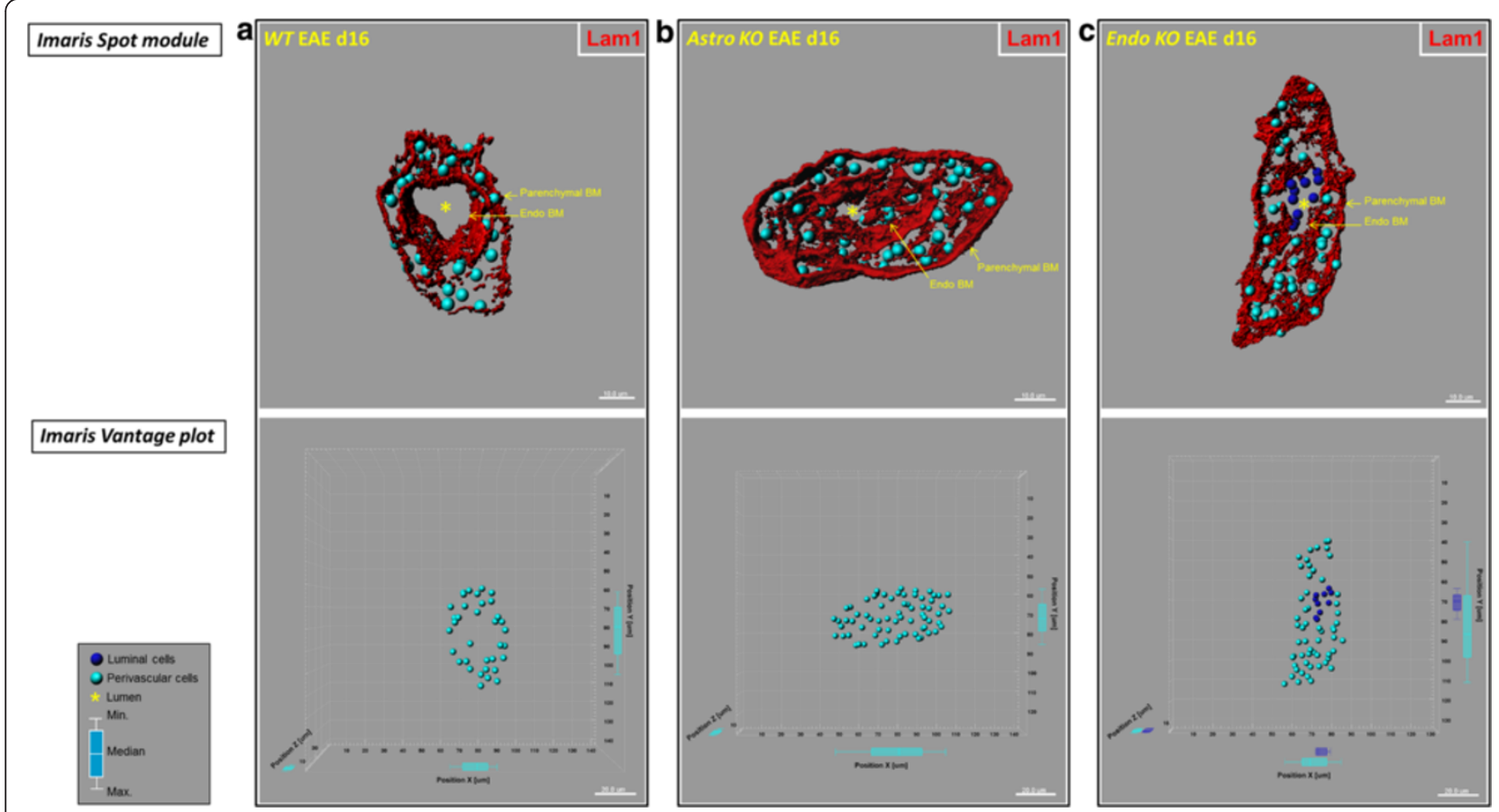

Figure 7 Astro $K O$ and Endo $K O$ mice display differences in 3D distribution profiles of luminal and perivascular cells. Isosurface-rendered images were generated from confocal z-stacks of 60- $\mu \mathrm{m}$ thick cryosections from WT, Astro KO, and Endo KO mice at d16 EAE. The BM is highlighted by staining of Lam1 (red). (Top row) DRAQ5 ${ }^{+}$nuclei in luminal and perivascular compartments were optically isolated using 3D contour based segmentation (as described in Materials and Methods), and pseudo-colored blue (luminal) and turquoise (perivascular), respectively. Using Imaris ${ }^{\oplus}$ spot creation module, each of these nuclei is shown in the 3D dataset (volume) as a "spot object," designating its luminal or perivascular location. Scale $=10 \mu \mathrm{m}$. (Bottom row) Imaris ${ }^{\oplus a n t a g e ~ p l o t s ~ s h o w i n g ~ t h e ~ 3 D ~ d i s t r i b u t i o n s ~ o f ~ l u m i n a l ~ a n d ~ p e r i v a s c u l a r ~ c e l l s ~ a l o n g ~ m i c r o v a s c u l a r ~} x, y$, and $z$-axes in the corresponding vessels from the top row. Scale $=20 \mu \mathrm{m}$. (a) Representative $W T$ vessel showing an empty lumen (*). (b) The lumen in the Astro $K O$ vessel also appears empty (*) but partially collapsed, possibly owing to accumulation of perivascular cells that are missing guidance cues from deleted astrocyte-derived CCL2. (c) In contrast, Endo KO vessel shows evidence of congregation of cells in the lumen (blue), possibly reflecting stalled leukocyte transmigration in absence of endothelial-derived CCL2. Box-and-whisker plots are shown indicating the maximum and minimum spread from the median, in $\mu \mathrm{m}$, of luminal or perivascular nuclei along the $x, y$, and $z$-axes.

consistent with the observed effects of CCL2 gene deletion being limited to the CNS. Further reflecting CNS action, Astro KO mice failed to show the parenchymal leukocyte infiltration and clear disruption of CLN-5 that accompanied WT EAE, while Endo KO mice revealed leukocytes apparently stalled in the lumen of spinal cord microvessels. Significantly, the combined effect of separate astrocyte and endothelial CCL2 elimination on clinical disease closely mirrors the phenotype reported when CCL2 gene ablation was confined to the "central compartment" by adoptive transfer of WT T cells [9] or transplantation of WT bone marrow [10] into global CCL2 KO mice; i.e., reduced disease severity along with delayed disease onset. This reinforces the notion that CCL2 from astrocytes and endothelial cells each contribute to EAE disease in a major, yet different way. The differences in EAE noted between Astro KO and Endo KO mice may reflect direct consequences of cell-specific CCL2 release on the CNS microvascular endothelium, leukocyte migration, or both. Possible actions of CCL2 released by CNS endothelial cells or astrocytes are schematized in Figure 8.

\section{Astrocyte-derived CCL2 regulates BBB integrity and leukocyte penetration into the CNS parenchyma, while endothelial-derived CCL2 impacts leukocyte transendothelial migration}

The failure of only Astro KO mice to show clear disruption of CLN-5 staining at d9 EAE supports the interpretation that CCL2 released from astrocytes figures prominently in destabilizing endothelial TJs at the abluminal microvascular surface early during the neuroinflammatory process [13]. Later loss of CLN-5 in these mice by d16 may, instead, reflect disruption of TJs by extravasating leukocytes. Astrocyte-derived CCL2 may additionally serve to recruit extravasated leukocytes into the parenchyma, as Astro KO mice revealed leukocytes congregated in the perivascular space. This latter role is supported by Carrillo-de Sauvage et al. [18], who described contact of CNS infiltrating $\mathrm{T}$ cells with 


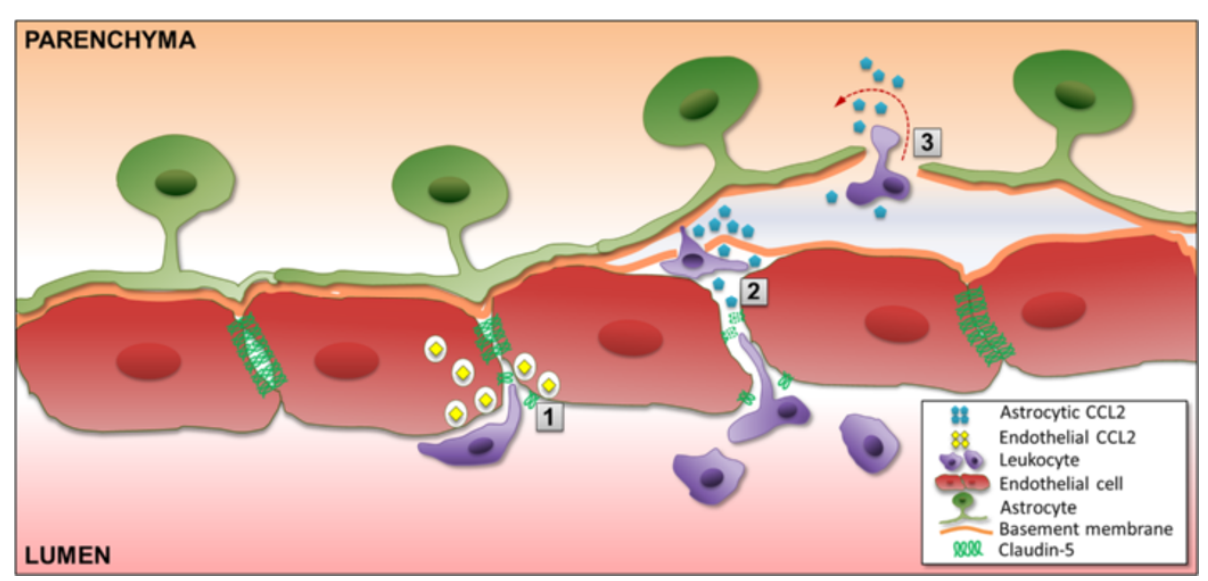

Figure 8 Differential actions of astrocyte-derived and endothelial cell-derived CCL2 at CNS venules. Based on observations with Astro KO and Endo $K O$ mice during EAE, the schematic depicts endothelial-derived CCL2 facilitating migration across the endothelium (1), a step post-adhesion. Astrocyte-derived CCL2 is shown promoting both breakdown of endothelial tight junctions (2), and penetration of leukocytes across the parenchymal BM into the CNS parenchyma (3).

CCL2-expressing perivascular astrocytes during neuroinflammatory disease.

The disruption of CLN-5-containing TJs during EAE and its prominent control by astrocyte-derived CCL2, is consistent with numerous reports on the action of CCL2 on TJs and BBB properties in cultured BMECs [13-15,40], CNS microvessels in vitro [13], and CNS microvessels in vivo in other neurological settings [41-43]. Notably, however, a recent report describing the effect of pertussis toxin injection in mice constitutively overexpressing CCL2 selectively in oligodendrocytes under direction of the myelin basic protein (MBP) promoter, found no evidence of a disrupted CLN-5 pattern accompanying leukocyte extravasation into brain [44]. This apparent contradiction may be due, in part, to the high level of chronic over-expression of CCL2 $(<100,000$ times normal values) having caused down-modulation of CCR2, the cognate receptor for CCL2, on BMECs [45], as well as inappropriate or inadequate access of oligodendrocytederived CCL2 - normally not found in health or disease to the CNS microvasculature. The present study thus underscores the unique relationship between endogenous, astrocyte-derived CCL2, TJs, and BBB permeability in neuroinflammatory disease [46].

Endothelial-derived CCL2, on the other hand, may facilitate a 'post-adhesion' stage of leukocyte extravasation, as the absence of this chemokine pool was uniquely associated with the appearance of leukocytes stalled within the microvascular lumen. In support of this hypothesis, Shulman et al. [23] recently showed that an intraendothelial vesicle pool of CCL2 within cultured HUVECs is a critical regulator of transendothelial migration of adherent effector T cells. Our data, showing both apparently stalled leukocytes in the microvascular lumen of Endo $K O$ mice and punctate CCL2 immunostaining in
BMECs of WT mice, may thus represent an extension of the results of Shulman et al. [23] to an in vivo scenario and advance a critical role for a CNS endothelial pool of CCL2, possibly vesicle bound, in mediating leukocyte transendothelial migration during neuroinflammatory disease. While this differs from the finding that CCL2 facilitates adhesion of leukocytes to pial microvessels [21], this distinction may represent the considerable endothelial heterogeneity that exists along the CNS microvasculature [47].

The smaller but significant loss of CLN-5 staining noted in Endo KO mice from d9 to d16 may chiefly represent the action of astrocyte-derived CCL2, as lesser extravasation was observed in these mice during this period. Conceivably, the astrocyte CCL2 pool could also have guided the lesser amount of extravasated cells into the parenchyma, resulting in the delayed disease noted.

\section{Cell-selective CCL2 knockout highlights CNS actions of CCL2 in neuroinflammatory disease}

Our collective findings reinforce critical and non-redundant roles of CNS CCL2 in mediating EAE, as previously implicated in adoptive transfer and bone marrow chimera EAE studies with global CCL2 knockout mice $[9,10]$. Of further importance, the use of Astro $K O$ and Endo $K O$ mice together with high-resolution $3 \mathrm{D}$ confocal imaging in this study was able to resolve apparently unique contributions of astrocyte and endothelial CCL2 pools to EAE pathogenesis. That both types of mice might share some effects of conditional CCL2 deletion is in accord with reports that BMECs can deposit CCL2 abluminally [48], and CCL2 can be transcytosed from the abluminal to luminal BMEC surface [34]. Because all endothelial cells in the Endo $K O$ mice are deficient in CCL2 expression, at this time it cannot be 
concluded that CCL2 from CNS endothelial cells, as opposed to peripheral endothelial cells, affected the disease process. However, given the failure of LNCs from these mice to show any deficits in MOG-stimulated proliferation, IFN- $\gamma$ or IL-17 production, or MOG MHC class II tetramer staining, it is doubtful that the absence of CCL2 from peripheral endothelial cells was a major factor in the aberrant EAE patterns noted. Diminished CLN-5 disruption and heightened presence of luminal leukocytes in Endo $K O$ mice further point to the CNS endothelial pool of CCL2 as featuring critically in EAE.

Though only EAE was analyzed in this study, astrocytes and BMECs have been suspected as critical sources of CCL2 during other neuroinflammatory conditions investigating three different CNS inflammatory scenarios (human glioma, striatal injection of LPS in mice, and adenovirally injected monkeys) reporting that extravasation of lymphocytes is mediated by CCL2-expressing astrocytes independent of the inflammatory situation and species [18]. Further, Tei et al. [49] most recently argued that CCL2 expression by both astrocytes and BMECs may contribute to the invasion and parenchymal migration of brain $\mathrm{Iba}^{+} / \mathrm{NG}^{+}$cells, descendants of subpopulations of circulating monocytes, following cerebral ischemia. Thus, expression of CCL2 by astrocytes and/or BMECs may be considered a widespread phenomenon associated with neuroinflammation. The seminal importance attributed to these particular sources of CCL2 does not preclude contributions by other CCL2-expressing cell types, e.g., microglia $[10,50]$, which may further modulate neuroinflammatory disease in their unique ways.

It has nevertheless been firmly established through elegant adoptive $\mathrm{T}$ cell transfer [9] and bone marrow chimera [10] studies, that CCL2 derived from the peripheral leukocyte compartment is not critical to the development of EAE. Hence, even though Tie-2-driven Cre expression has been reported in cells of the hematopoietic lineage [51], any potential loss of CCL2 from this population would not detract from our interpretation that CCL2 elimination from BMEC, rather than leukocytes, predominantly altered EAE disease. The delay of disease phenotype in Endo $K O$ mice, in fact, is consistent with the lack of CCL2 immunostaining in BMEC and apparent stalling of adherent leukocytes observed in these animals.

\section{Therapeutic targeting of CNS CCL2}

Lastly, as the BBB has generally been recognized as the major impediment to drug delivery to the CNS [52], these results have significant implications for targeting CCL2 in the treatment of neuroinflammatory disease. It is thus notable to point out that injection of a CCL2neutralizing antibody directly into the brain was effective at suppressing lymphocyte infiltration following striatal lipopolysaccharide injection [18], a situation in which astrocytes were observed to be the major CCL2expressing cell type. Arguably, therapeutic inhibition of endothelial CCL2 would not require circumventing or penetrating the $\mathrm{BBB}$, in contrast to suppressing astrocyte production of this chemokine. However, as our and the recent results of Shulman et al. [23] point out, merely targeting CCL2 with antibodies or receptor antagonists may not be effective against vesicle-bound endothelial CCL2 depots. The recent demonstration that the antiinflammatory compound bindarit, a synthetic indazolic derivative (MWr 324 Daltons) that preferentially inhibits transcription of the monocyte chemoattractant subfamily of CC chemokines (MCP-1/CCL2, MCP-2/CCL8, and MCP-3/CCL7), delayed and suppressed EAE in concert with diminishing CNS microvascular CCL2 expression [33], suggests that interfering with intra-endothelial CCL2 might be of high therapeutic value. However, as BBB disruption often accompanies neuroinflammatory disease [30,47], drugs that inhibit CCL2 synthesis could potentially have opportunity to strike at both endothelial cells and astrocytes, even if only with limited efficiency at the latter, and thus offer better therapeutic prospects than antibodies or antagonists. Recent descriptions of CCL2 involvement in post-ischemic disruption of the BBB [43], beta-amyloid neurotoxicity [53], and traumatic brain injury [54], further underscore that modulating CNS CCL2 synthesis at the vascular and/or parenchymal level may offer a novel therapeutic option for a wide range of neuropathologies.

\section{Conclusions}

In light of our results, it is determined that CCL2 from either astrocytes or BMECs separately impacts clinical $\mathrm{EAE}$ and associated neuroinflammatory processes in distinct ways and through different mechanisms depending on the source cell type. CCL2 from astrocytes regulates severity of clinical EAE disease, while controlling penetration of leukocytes into the CNS parenchyma and disrupting CLN-5 staining pattern along the CNS microvasculature. In contrast, CCL2 from BMECs appears to more so determine disease onset, and effect post-adhesion leukocyte transendothelial migration. Therapeutic targeting of CCL2 expression or action at the parenchymal and/or vascular levels may thus offer promise in treating neuroinflammatory disease.

\section{Additional files}

Additional file 1: Contour-based 3D segmentation of luminal- and perivascular-associated cells in Endo $K O$ mice during EAE. The video shows $3 \mathrm{D}$ reconstruction of the representative Endo $K O$ venule from Figure 7, allowing for enhanced visualization of the perivascular and luminal cellularity associated with the microvessel along $x, y$, and $z$ axes. It further demonstrates the sequential steps employed for the contour-based 3D segmentation of DRAQ5 $5^{+}$cells into separate luminal 
and perivascular compartments, using 3D contour surfaces, as described in Materials and Methods. The nuclei were pseudo-colored in blue (luminal) and turquoise (perivascular). Each $180^{\circ}$-turn in the video indicates the following steps in sequence - 3D reconstruction $\rightarrow$ Lam1 (Red) isosurface $\rightarrow$ parenchymal BM contour $\rightarrow$ endothelial BM contour $\rightarrow$ segmented nuclei.

Additional file 2: Figure S1. Specificity of CCL2 immunostaining. Volume rendered images of $z$-stacks obtained from serialsections of $d 9$ EAE spinal cords used in Figure 6 (left) and naive (right) mice demonstrating specific immunoreactivity of the CCL2 antibody. No detectable CCL2 staining (green) was observed in naïve mice upon incubation with CCL2 antibody or in EAE mice in the absence of primary antibody. The endothelium is highlighted with CD31 (red), while DRAQ5 staining reveals the nuclei (blue). Scale $=20 \mu \mathrm{m}$

Additional file 3: Figure S2. Lack of focal CLN-5 immunostaining loss and perivascular cellularity in naïve spinal microvessels. Isosurface-rendered images generated from confocal $z$-stacks of 60 -um thick cryosections from naïve mice showing continuity of CLN-5 staining (green) in naïve spinal microvessels. The lack of perivascular cellularity associated with typical inflamed microvessels is further highlighted with DRAQ5 staining for nuclei (blue). Scale $=20 \mu \mathrm{m}$.

\section{Abbreviations}

Astro KO: Astrocyte-specific CCL2 knockout mice; BBB: Blood-brain barrier BMEC: Brain microvascular endothelial cells; BM: Basement membrane; CCL2: Chemokine (C-C motif) ligand 2; CFSE: Carboxyfluorescein succinimidyl ester; CLN-5: Claudin-5; CNS: Central nervous system; EAE: Experimental autoimmune encephalomyelitis; Endo KO: Endothelial-specific CCL2 knockout mice; FACS: Fluorescence-activated cell sorting; GFAP: Glial fibrillary acid protein; HUVECs: Human umbilical vein endothelial cells; IL: Interleukin; Immuno-EM: Immuno-electron microscopy; Lam 1: Laminin 1; LNC: Lymph node cells: MHC: Major histocompatibility complex: MOG: Myelin oligodendrocyte glycoprotein; MS: Multiple sclerosis; PB: Phosphate buffer PBS: Phosphate buffered saline; TJ: Tight junction; WT: Wild type mice.

\section{Competing interests}

The authors declare that they have no competing interests.

\section{Authors' contributions}

JSP, SG, and DP designed the experiments and wrote the paper. DP, SG, and YL performed the experiments. ERJ assisted with LNC proliferation and FACS analysis. DRS acquired the EM images and NHR provided instruction with the EAE model. All authors read and approved the final manuscript.

\section{Acknowledgements}

This work was supported in part by grant R01-MH061525 from the National Institutes of Health, and grants PP-1215 and RG 4828-A-5 from the National Multiple Sclerosis Society, to JSP. The authors are grateful to Dr. Robert Cone for helpful discussions.

\section{Author details}

'Department of Cell Biology, Blood-brain Barrier Laboratory, 263 Farmington Ave., Farmington CT 06030, USA. ${ }^{2}$ Department of Immunology, University of Connecticut Health Center, Farmington CT 06030, USA. ${ }^{3}$ Department of Physiology and Neurobiology, University of Connecticut, Storrs CT 06269, USA. ${ }^{4}$ School of Public Health, Yale University, New Haven CT 06520, USA.

Received: 31 October 2013 Accepted: 28 December 2013 Published: 21 January 2014

\section{References}

1. Leonard EJ, Skeel A, Yoshimura T: Biological aspects of monocyte chemoattractant protein-1 (MCP-1). Adv Exp Med Biol 1991, 305:57-64.

2. Mantovani A, Sozzani S, Bottazzi B, Peri G, Sciacca FL, Locati M, Colotta F: Monocyte chemotactic protein-1 (MCP-1): signal transduction and involvement in the regulation of macrophage traffic in normal and neoplastic tissues. Adv Exp Med Biol 1993, 351:47-54.

3. Bennett JL, Elhofy A, Canto MC, Tani M, Ransohoff RM, Karpus WJ: CCL2 transgene expression in the central nervous system directs diffuse infiltration of CD45(high)CD11b(+) monocytes and enhanced
Theiler's murine encephalomyelitis virus-induced demyelinating disease. J Neurovirol 2003, 9:623-636.

4. Toft-Hansen H, Buist R, Sun XJ, Schellenberg A, Peeling J, Owens T: Metalloproteinases control brain inflammation induced by pertussis toxin in mice overexpressing the chemokine CCL2 in the central nervous system. J Immunol 2006, 177:7242-7249.

5. Yadav A, Saini V, Arora S: MCP-1: chemoattractant with a role beyond immunity: a review. Clin Chim Acta 2010, 411:1570-1579.

6. Izikson L, Klein RS, Luster AD, Weiner HL: Targeting monocyte recruitment in CNS autoimmune disease. Clin Immunol 2002, 103:125-131.

7. Mahad DJ, Ransohoff RM: The role of MCP-1 (CCL2) and CCR2 in multiple sclerosis and experimental autoimmune encephalomyelitis (EAE). Semin Immunol 2003, 15:23-32.

8. Conductier G, Blondeau N, Guyon A, Nahon JL, Rovere C: The role of monocyte chemoattractant protein MCP1/CCL2 in neuroinflammatory diseases. J Neuroimmunol 2010, 224:93-100

9. Huang DR, Wang J, Kivisakk P, Rollins BJ, Ransohoff RM: Absence of monocyte chemoattractant protein 1 in mice leads to decreased local macrophage recruitment and antigen-specific $\mathrm{T}$ helper cell type 1 immune response in experimental autoimmune encephalomyelitis. J Exp Med 2001, 193:713-726.

10. Dogan RN, Elhofy A, Karpus WJ: Production of CCL2 by central nervous system cells regulates development of murine experimental autoimmune encephalomyelitis through the recruitment of TNF- and iNOS-expressing macrophages and myeloid dendritic cells. J Immunol 2008, 180:7376-7384

11. Giraud SN, Caron CM, Pham-Dinh D, Kitabgi P, Nicot AB: Estradiol inhibits ongoing autoimmune neuroinflammation and NFkappaB-dependent CCL2 expression in reactive astrocytes. Proc Natl Acad Sci USA 2010, 107:8416-8421.

12. Hermann DM, EIAli A: The abluminal endothelial membrane in neurovascular remodeling in health and disease. Sci Signal 2012, 5:re4.

13. Song L, Pachter JS: Monocyte chemoattractant protein-1 alters expression of tight junction-associated proteins in brain microvascular endothelial cells. Microvasc Res 2004, 67:78-89.

14. Stamatovic SM, Keep RF, Kunkel SL, Andjelkovic AV: Potential role of MCP-1 in endothelial cell tight junction 'opening': signaling via Rho and Rho kinase. J Cell Sci 2003, 116:4615-4628.

15. Yao Y, Tsirka SE: Truncation of monocyte chemoattractant protein 1 by plasmin promotes blood-brain barrier disruption. J Cell Sci 2011, 124:1486-1495.

16. Bennett J, Basivireddy J, Kollar A, Biron KE, Reickmann P, Jefferies WA, McQuaid S: Blood-brain barrier disruption and enhanced vascular permeability in the multiple sclerosis model EAE. J Neuroimmunol 2010, 229:180-191.

17. Meinl E, Krumbholz M, Derfuss T, Junker A, Hohlfeld R: Compartmentalization of inflammation in the CNS: a major mechanism driving progressive multiple sclerosis. J Neurol Sci 2008, 274:42-44.

18. Carrillo-de Sauvage MA, Gomez A, Ros CM, Ros-Bernal F, Martin ED, Perez-Valles A, Gallego-Sanchez JM, Fernandez-Villalba E, Barcia C Sr, Barcia C Jr, Herrero MT: CCL2-expressing astrocytes mediate the extravasation of T lymphocytes in the brain. Evidence from patients with glioma and experimental models in vivo. PLoS One 2012, 7:e30762.

19. Berman JW, Guida MP, Warren J, Amat J, Brosnan CF: Localization of monocyte chemoattractant peptide-1 expression in the central nervous system in experimental autoimmune encephalomyelitis and trauma in the rat. J Immunol 1996, 156:3017-3023.

20. Subileau EA, Rezaie P, Davies HA, Colyer FM, Greenwood J, Male DK, Romero IA: Expression of chemokines and their receptors by human brain endothelium: implications for multiple sclerosis. J Neuropathol Exp Neurol 2009, 68:227-240.

21. dos Santos AC, Barsante MM, Arantes RM, Bernard CC, Teixeira MM, Carvalho-Tavares J: CCL2 and CCL5 mediate leukocyte adhesion in experimental autoimmune encephalomyelitis-an intravital microscopy study. J Neuroimmunol 2005, 162:122-129.

22. Seguin R, Biernacki K, Rotondo RL, Prat A, Antel JP: Regulation and functional effects of monocyte migration across human brain-derived endothelial cells. J Neuropathol Exp Neurol 2003, 62:412-419.

23. Shulman Z, Cohen SJ, Roediger B, Kalchenko V, Jain R, Grabovsky V, Klein E, Shinder V, Stoler-Barak L, Feigelson SW, Meshel T, Nurmi SM, Goldstein I, Hartley O, Gahmberg CG, Etzioni A, Weninger W, Ben-Baruch A, Alon R: 
Transendothelial migration of lymphocytes mediated by intraendothelial vesicle stores rather than by extracellular chemokine depots. Nat Immunol 2012, 13:67-76.

24. Ge S, Murugesan N, Pachter JS: Astrocyte- and endothelial-targeted CCL2 conditional knockout mice: critical tools for studying the pathogenesis of neuroinflammation. J Mol Neurosci 2009, 39:269-283.

25. Murugesan N, Paul D, Lemire Y, Shrestha B, Ge S, Pachter JS: Active induction of experimental autoimmune encephalomyelitis by MOG35-55 peptide immunization is associated with differential responses in separate compartments of the choroid plexus. Fluids Barriers CNS 2012, 9:15-31.

26. Goverman J, Brabb T, Paez A, Harrington C, von Dassow P: Initiation and regulation of CNS autoimmunity. Crit Rev Immunol 1997, 17:469-480.

27. Racke MK, Hu W, Lovett-Racke AE: PTX cruiser: driving autoimmunity via TLR4. Trends Immunol 2005, 26:289-291.

28. Suen WE, Bergman CM, Hjelmstrom P, Ruddle NH: A critical role for lymphotoxin in experimental allergic encephalomyelitis. J Exp Med 1997 186:1233-1240.

29. Cravens PD, Hussain RZ, Zacharias TE, Ben LH, Herndon E, Vinnakota R, Lambracht-Washington D, Nessler S, Zamvil SS, Eagar TN, Stuve O: Lymph node-derived donor encephalitogenic CD4+ T cells in C57BL/6 mice adoptive transfer experimental autoimmune encephalomyelitis highly express GM-CSF and T-bet. J Neuroinflammation 2011, 8:73-87.

30. Paul D, Cowan AE, Ge S, Pachter JS: Novel 3D analysis of Claudin-5 reveals significant endothelial heterogeneity among CNS microvessels. MicrovasC Res 2013, 86:1-10.

31. Owens T, Bechmann I, Engelhardt B: Perivascular spaces and the two steps to neuroinflammation. J Neuropathol Exp Neurol 2008, 67:1113-1121.

32. Li Y, Serwanski DR, Miralles CP, Fiondella CG, Loturco JJ, Rubio ME, De Blas $\mathrm{AL}$ : Synaptic and nonsynaptic localization of protocadherin-gammaC5 in the rat brain. J Comp Neurol 2010, 518:3439-3463.

33. Ge S, Shrestha B, Paul D, Keating C, Cone R, Guglielmotti A, Pachter JS: The CCL2 synthesis inhibitor bindarit targets cells of the neurovascular unit, and suppresses experimental autoimmune encephalomyelitis. J Neuroinflammation 2012, 9:17-184.

34. Ge S, Song L, Serwanski DR, Kuziel WA, Pachter JS: Transcellular transport of CCL2 across brain microvascular endothelial cells. J Neurochem 2008, 104:1219-1232.

35. Palframan RT, Jung S, Cheng G, Weninger W, Luo Y, Dorf M, Littman DR, Rollins BJ, Zweerink H, Rot A, von Andrian UH: Inflammatory chemokine transport and presentation in HEV: a remote control mechanism for monocyte recruitment to lymph nodes in inflamed tissues. J Exp Med 2001, 194:1361-1373.

36. Jimenez F, Quinones MP, Martinez HG, Estrada CA, Clark K, Garavito E, Ibarra J, Melby PC, Ahuja SS: CCR2 plays a critical role in dendritic cell maturation: possible role of CCL2 and NF-kappa B. J Immunol 2010, 184:5571-5581.

37. Dal Secco V, Soldani C, Debrat C, Asperti-Boursin F, Donnadieu E, Viola A Sarukhan A: Tunable chemokine production by antigen presenting dendritic cells in response to changes in regulatory T cell frequency in mouse reactive lymph nodes. PLOS One 2009, 4:e7696.

38. Damsker JM, Hansen AM, Caspi RR: Th1 and Th17 cells: adversaries and collaborators. Ann N Y Acad Sci 2010, 1183:211-221.

39. Nitta T, Hata M, Gotoh S, Seo Y, Sasaki H, Hashimoto N, Furuse M, Tsukita S: Size-selective loosening of the blood-brain barrier in claudin-5-deficient mice. J Cell Biol 2003, 161:653-660.

40. Dhillon NK, Peng F, Bokhari S, Callen S, Shin SH, Zhu X, Kim KJ, Buch SJ: Cocaine-mediated alteration in tight junction protein expression and modulation of CCL2/CCR2 axis across the blood-brain barrier: implications for HIV-dementia. J Neuroimmune Pharmacol 2008, 3:52-56.

41. Stamatovic SM, Shakui P, Keep RF, Moore BB, Kunkel SL, Van Rooijen N Andjelkovic AV: Monocyte chemoattractant protein-1 regulation of blood-brain barrier permeability. J Cereb Blood Flow Metab 2005, 25:593-606.

42. Dimitrijevic OB, Stamatovic SM, Keep RF, Andjelkovic AV: Effects of the chemokine CCL2 on blood-brain barrier permeability during ischemia-reperfusion injury. J Cereb Blood Flow Metab 2006, 26:797-810.

43. Strecker JK, Minnerup J, Schutte-Nutgen K, Gess B, Schabitz WR, Schilling M: Monocyte chemoattractant protein-1-deficiency results in altered blood-brain barrier breakdown after experimental stroke. Stroke 2013, 44:2536-2544.

44. Schellenberg AE, Buist R, Del Bigio MR, Toft-Hansen H, Khorooshi R, Owens T, Peeling J: Blood-brain barrier disruption in CCL2 transgenic mice during pertussis toxin-induced brain inflammation. Fluids Barriers CNS 2012, 9:10-19.

45. Dzenko KA, Andjelkovic AV, Kuziel WA, Pachter JS: The chemokine receptor CCR2 mediates the binding and internalization of monocyte chemoattractant protein-1 along brain microvessels. J Neurosci 2001 21:9214-9223.

46. Yao Y, Tsirka SE: Monocyte chemoattractant protein-1 and the blood-brain barrier. Cell Mol Life Sci 2013 [Ahead of print].

47. Ge S, Song L, Pachter JS: Where is the blood-brain barrier ... really? J Neurosci Res 2005, 79:421-427.

48. Chui R, Dorovini-Zis K: Regulation of CCL2 and CCL3 expression in human brain endothelial cells by cytokines and lipopolysaccharide. J Neuroinflammation 2010, 7:1.

49. Tei N, Tanaka J, Sugimoto K, Nishihara T, Nishioka R, Takahashi H, Yano H, Matsumoto S, Ohue S, Watanabe H, Kumon Y, Ohnishi T: Expression of MCP-1 and fractalkine on endothelial cells and astrocytes may contribute to the invasion and migration of brain macrophages in ischemic rat brain lesions. J Neurosci Res 2013, 91:681-693.

50. Starossom SC, Mascanfroni ID, Imitola J, Cao L, Raddassi K, Hernandez SF, Bassil R, Croci DO, Cerliani JP, Delacour D, Wang Y, Elyaman W, Khoury SJ, Rabinovich GA: Galectin-1 deactivates classically activated microglia and protects from inflammation-induced neurodegeneration. Immunity 2012, 37:249-263.

51. Tang $Y$, Harrington $A$, Yang $X$, Friesel RE, Liaw $L$ : The contribution of the Tie2+ lineage to primitive and definitive hematopoietic cells. Genesis 2010, 48:563-567.

52. Pardridge WM: The blood-brain barrier: bottleneck in brain drug development. NeuroRx 2005, 2:3-14.

53. Severini C, Passeri PP, Ciotti M, Florenzano F, Possenti R, Zona C, Di Matteo A, Guglielmotti A, Calissano P, Pachter J, Mercanti D: Bindarit, inhibitor of CCL2 synthesis, protects neurons against amyloid-beta-induced toxicity. J Alzheimers Dis 2014, 38(2):281-293.

54. Ho L, Zhao W, Dams-O'Connor K, Tang CY, Gordon W, Peskind ER, Yemul S, Haroutunian V, Pasinetti GM: Elevated plasma MCP-1 concentration following traumatic brain injury as a potential "predisposition" factor associated with an increased risk for subsequent development of Alzheimer's disease. J Alzheimers Dis 2012, 31:301-313.

doi:10.1186/1742-2094-11-10

Cite this article as: Paul et al:: Cell-selective knockout and 3D confocal image analysis reveals separate roles for astrocyte-and endothelialderived CCL2 in neuroinflammation. Journal of Neuroinflammation 2014 11:10

\section{Submit your next manuscript to BioMed Central and take full advantage of:}

- Convenient online submission

- Thorough peer review

- No space constraints or color figure charges

- Immediate publication on acceptance

- Inclusion in PubMed, CAS, Scopus and Google Scholar

- Research which is freely available for redistribution 